Itinéraires Itinéraires

Littérature, textes, cultures

2020-3 | 2021

Le rap, une poésie de performances

\title{
Et les rappeurs chantèrent Senghor ou un exemple d'adaptation intermédiale de la négritude
}

And the Rap Artists Sang Senghor or an Example of Intermedial Adaptation of Negritude

Serigne Seye

\section{CpenEdition}

Journals

Édition électronique

URL : https://journals.openedition.org/itineraires/9392

DOI : $10.4000 /$ itineraires.9392

ISSN : 2427-920X

Éditeur

Pléiade

Référence électronique

Serigne Seye, «Et les rappeurs chantèrent Senghor ou un exemple d'adaptation intermédiale de la négritude », Itinéraires [En ligne], 2020-3 | 2021, mis en ligne le 10 décembre 2021, consulté le 12 décembre 2021. URL : http://journals.openedition.org/itineraires/9392 ; DOI : https://doi.org/10.4000/ itineraires.9392

Ce document a été généré automatiquement le 12 décembre 2021.

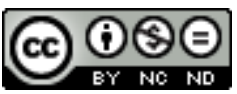

Itinéraires est mis à disposition selon les termes de la licence Creative Commons Attribution - Pas d'Utilisation Commerciale - Pas de Modification 4.0 International. 
Et les rappeurs chantèrent Senghor ou un exemple d'adaptation intermédiale de la négritude

And the Rap Artists Sang Senghor or an Example of Intermedial Adaptation of Negritude

Serigne Seye

Introduction 
Figure 1. Senghor, figure majeure de la négritude

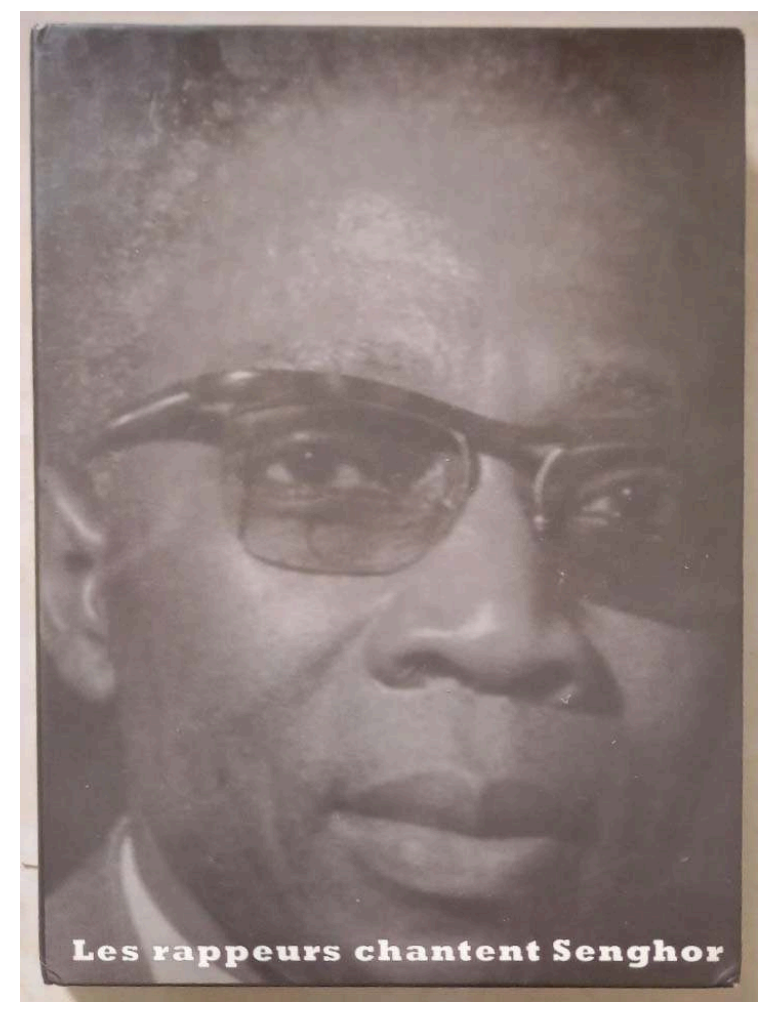

Crédits : Medialab/Waru Studio

1 Définie par son principal théoricien Jürgen Müller comme « le fait qu'un média recèle en soi des structures et des possibilités d'un ou de plusieurs autres médias » (2016: 100), l'intermédialité nous paraît être l'approche la plus pertinente dans le domaine littéraire pour étudier les relations d'influence et de contamination réciproques que partagent la littérature et la musique. Cela est d'autant plus vrai que l'œuvre que nous allons analyser fait appel à plusieurs médiums pour se construire. Elle est en effet constituée d'un coffret regroupant un livre, un CD et un DVD, dont les poèmes de Senghor et les rappeurs qui les déclament demeurent le trait d'union. Cette production (Les rappeurs chantent Senghor), parue en 2007, s'inscrivait dans un contexte de célébration du centenaire de la naissance de Senghor en $2006^{1}$. Les autorités étatiques sénégalaises voulaient que cette année coïncidât avec la redécouverte, par les artistes et hommes de lettres, de l'œuvre du président poète. C'est dans cette perspective que Safouane Pindra, manager général du label dakarois Optimiste Produktions ${ }^{2}$, réunit huit groupes et rappeurs en solo originaires de cinq pays d'Afrique noire francophone (Dhalai-K du Bénin, Yeleen du Burkina Faso, Boudor du Cameroun, Lord Ekomy Ndong du Gabon et Keyti, Xuman, Wabmg 44, Lyrical Ceddo du Sénégal) autour de l'œuvre du président-poète dans le but, selon le producteur, "d'investir les médiathèques de l'Afrique afin que ses jeunes puissent découvrir la pensée senghorienne ${ }^{3}$ ».

2 Cette association, qui peut paraître contre-nature, nous pousse à nous demander comment le rap peut être un lieu de re-médiatisation d'une œuvre originellement littéraire et aussi un espace idéal de transmédialité permettant de remettre au goût du jour des classiques de la littérature écrite ${ }^{4}$.

Dans notre travail, nous essaierons d'analyser la manière dont la chanson de rap accueille le poème senghorien, le transforme et/ou se laisse influencer par celui-ci. En 
convoquant les idées développées sur l'intermédialité et la transmédialité, nous allons voir comment se fait le transfert du poème d'un médium à l'autre, et quels sont les changements induits par cette adaptation musicale d'un chef-d'œuvre de la poésie négro-africaine ${ }^{5}$. La transmédialité, comprise comme «la capacité d'une œuvre à se développer en dehors d'un unique média» (Krajewski 2015: §14) ou encore «le processus dans lequel les éléments d'une fiction sont dispersés sur diverses plateformes médiatiques dans le but de créer une expérience de divertissement coordonnée et unifiée ${ }^{6}$ " (Jenkins 2006 : 95-96), nous montrera l'adaptabilité simultanée à différents médias d'œuvres littéraires africaines qui n'étaient pas initialement programmées pour un tel destin. En même temps, associée à l'intermédialité, cette approche nous aidera à présenter la musique et le texte de rap comme un lieu de recréation et de recyclage de différents objets 7 . Les vers de Senghor, en effet, se déploient hors du média littérature et du médium que constitue le livre pour se réactualiser, se remédiatiser dans une œuvre musicale d'abord (dans un CD) puis visuelle. Nous nous limitons dans cette analyse au premier transfert, celui du recueil au CD en passant par le livret. L'approche adoptée permet aussi d'étendre le concept de transmédialité à un corpus souvent marginalisé au profit d'objets narratifs purs construits dans le cadre du cinéma et des jeux vidéo.

\section{Les motivations artistiques d'une reprise intermédiale}

4 Ce coffret participe à la conservation et à la valorisation d'un patrimoine littéraire qui semble parfois limité à certains cercles intellectuels. Les rappeurs aident à le populariser auprès d'une certaine jeunesse et à l'introduire dans un milieu dont les membres souffrent de préjugés les présentant comme des individus peu cultivés. Si Senghor, l'homme politique, n'est pas forcément apprécié des rappeurs africains, il en est autrement du poète, qui est considéré comme une grande figure de la création littéraire et un véritable chantre des valeurs de la négritude. De manière générale, on note une certaine proximité des MCs sénégalais et africains avec la négritude, qu'Abdoulaye Niang (2011) appelle à dépasser. Ce qui ne peut se faire qu'après un travail préalable sur le patrimoine artistique légué par ce mouvement. C'est peut-être ce qui explique la participation des rappeurs qui, comme Keyti, se réapproprient les poèmes de Senghor et leur font subir un réaménagement, parce que la fascination pour cet écrivain n'empêche pas une transgression, timide mais pourtant réelle, qui affirme encore la puissance créatrice du MC et sa liberté. Ce travail de réajustement peut prendre la forme d'une transformation de l'hypotexte senghorien en un hypertexte ou celle d'une simple reprise. Le but du rappeur étant de ne pas laisser le poème de Senghor intact, il veut le refaire à son goût et a pour cela différentes possibilités : il peut choisir d'agir sur les mots, le rythme, la structure ou les trois à la fois. C'est ce type de travail de subjectivation qui fait dire à Christophe Kihm: "Quels que soient ses moyens, la nécessité de revivre et/ou de refaire, propre à la reprise, a pour effet de soumettre l'événement premier à l'altération et au brouillage de ses données constitutives par retraits, ajouts et interférences » (Kihm $2010: 26$ ).

5 Le mot est donc lâché : cette adaptation intermédiale qui apparaît dans le titre de cet article est donc une sorte de reprise dans la mesure où les MCs reprennent et réinterprètent une œuvre qui a été composée et "jouée " par le truchement de l'écriture. C'est un acte rétroactif permettant le retour sur un objet - un événement 
selon Kihm - et la mise en relation de deux médias dont l'un est la réactualisation de l'autre. Nous avons donc utilisé le concept de reprise dans le sens où l'entend Kihm :

Expérience qui réalise la mise en force de deux événements (linguistiques, sociaux, culturels, sonores, etc.), le premier reconnu comme point d'origine et le second comme point d'actualisation du premier par l'opération et la mise en acte d'une répétition. Toute reprise est active et rétroactive, et se détermine dans les distances et les écarts produits entre un point d'origine et son actualisation. Toute reprise est le produit, mais aussi la marque d'une expérience de subjectivation. (Kihm 2010: 25)

6 Ainsi, tout en faisant un travail qui se trouve en même temps être une expérience de subjectivation, les rappeurs de ce projet s'adonnent à une duplication de l'œuvre senghorienne qui entretient avec le premier événement, c'est-à-dire le média d'origine, des relations ambiguës de ressemblance/dissemblance. Cela ne fait pas moins de leurs productions des versions actualisées et modernisées de la poésie de Senghor. Les propos de Kihm abondent dans le même sens: «la reprise saisit la totalité d'un événement, mais ne le restitue jamais à l'identique. Une prise (toujours partielle) permet donc à une totalité d'être restituée différemment (toujours partiale) plus proche d'elle sur le plan chronologique » (Kihm 2010 : 26). La nouvelle œuvre, obtenue après le travail de reprise, est ainsi désignée par Kihm sous les noms d'objet ( $\left.a^{\prime}\right)$, d'épreuve ou d'actualisation, alors que l'œuvre de départ est appelée objet (a), original ou modèle. Ce dernier est soumis, par les rappeurs, à un travail de création portant aussi bien sur la textualité que sur la musicalité et la mise en images du texte. Ils nous prouvent que le même média, décontextualisé et remédiatisé, n'est jamais le même. La mise en présence de deux médias suppose un jeu de traduction mutuelle, de translation et de transcription de l'un par l'autre. Le rappeur joue donc un rôle de médiateur, de passeur de frontières médiatiques dont le travail relie les objets (a) et (a') dans le but de construire un hypermédia qui condense en son sein le littéraire et le musical pour postuler à l'intermédialité.

$7 \quad$ Les vers de Senghor se déploient hors du média littérature et du médium que constitue le livre pour se réactualiser, se remédiatiser dans l'œuvre musicale. Sur le plan chronologique, il est possible d'identifier les différentes productions médiatiques car l'œuvre, dans un premier temps, a d'abord existé en tant qu'ensemble de poèmes édité. Elle a ensuite été adaptée musicalement et chantée dans un second temps. La mise en vidéo ne vient qu'en dernière position pour achever le processus de double transfert. Le transfert consiste dans le cadre des recherches intermédiales en un «transport de matériaux ou de technologies [...] d'un média à un autre» (Jambrina 2015). Ce changement de contexte médiatique provoque inévitablement une transformation intrinsèque des éléments transportés, le même prenant parfois la forme d'autre chose. C'est ce que confirme Silvestra Mariniello quand elle avance que «le matériau et la technologie transportés se transforment dans le processus puisque leur identité et leurs sens sont fonction d'une relation à un contexte » $(2010: 13)$. De ce fait, les poèmes de Senghor, adaptés et transférés dans le média musical et visuel, épousent de nouvelles structures et s'offrent un supplément de sens dû à leur transfert. L'œuvre remédiatisée, ici les poèmes de Senghor, est supplantée par le nouveau qui, par sa plus grande adaptabilité au contexte contemporain, parvient à impacter, non seulement le sens et la forme de l'hypomédia, mais aussi sa réception. Celle-ci prend en charge en même temps le média d'origine et l'hypermédia le recyclant. Concrètement, cela signifie que l'auditeur ou le téléspectateur (concernant le DVD, qui n'est pas ici analysé) 
n'est pas seulement en contact avec une musique rap et un clip vidéo, mais tout cela et les poèmes de Senghor en même temps. C'est ce qui fait du coffret une véritable œuvre synthétique.

8 L'intermédialité ne se limite ainsi pas seulement au transfert d'éléments d'un média à l'autre. Dans le média d'accueil ou hypermédia, les contenus et les formes transférés sont retravaillés et inscrits dans une intermédialité interne qui donne l'occasion de réaliser un travail très abouti, dont le but est de créer une mémoire visuelle et iconographique.

\section{Lecture intermédiale du " paramédia » : une omniprésence de la figure de Senghor}

Figure 2. Le paramédia, version intermédiale du paratexte, mime l'écriture journalistique

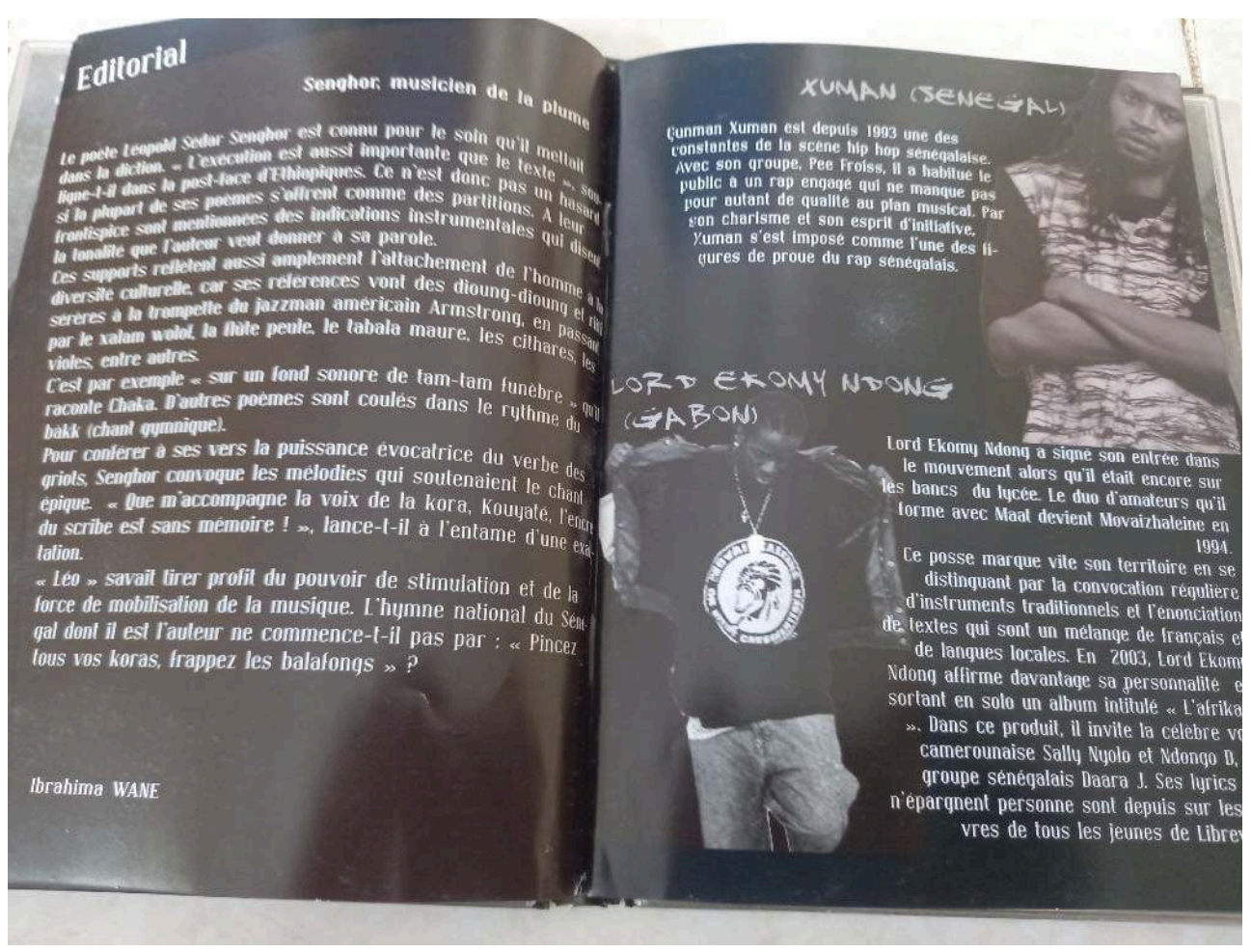

Crédits : Medialab/Waru Studio.

9 Tel que nous l'avons conçu, le paramédia est pour tout média non livresque ce que le paratexte est pour le texte. Ce néologisme désigne alors, pour nous, l'ensemble des éléments écrits et visuels qui encadrent un média. Par exemple, les éléments qui se trouvent sur la jaquette d'un CD ou d'une cassette : le titre, le nom du chanteur et du groupe, les images, les renseignements fournis au verso de la jaquette, etc. L'analyse du paramédia de l'œuvre Les rappeurs chantent Senghor (2007) révèle de prime abord son caractère intermédiatique parce que s'y côtoient écriture, image, références à la littérature et à la musique.

Cette intermédialité du paramédia se manifeste, tout d'abord, par le rapprochement entre le nom de Léopold Sédar Senghor et le groupe nominal "les rappeurs » qui semble relever, a priori, de l'anachronisme et même de l'antithèse. Le premier évoluant 
dans un univers qui peut sembler en déphasage avec une musique comme le rap et ayant écrit l'essentiel de son œuvre poétique à un moment où ce genre musical n'existait pas encore et où le mouvement hip-hop en était à ses balbutiements. À l'époque où, en effet, le hip-hop commence à sortir du Bronx pour se propager dans les autres quartiers de New York d'abord, puis dans les autres villes des États-Unis, Senghor était président du Sénégal depuis dix-neuf ans et avait déjà publié l'essentiel de sa production littéraire. Il n'y avait donc apparemment aucune raison pour que sur le plan historique Senghor rencontrât le rap. Cependant, son attachement à la culture afro-américaine, et au jazz en particulier, nous laisse croire que la rencontre aurait été inévitable si l'essor du mouvement hip-hop avait coïncidé avec le temps où il publiait des textes théoriques et des recueils de poèmes. Son goût pour la déclamation du texte poétique et ses fameuses performances de tassu ${ }^{8}$ lors d'entretiens télévisés démontrent une proximité avant l'heure avec ce genre musical. De plus, dans le recueil Éthiopiques ${ }^{9}$ (Senghor 1990), il a consacré un texte à la ville de New York en insistant sur l'aspect africain du quartier de Harlem, aujourd'hui l'un des bastions du hip-hop américain. La musique et la chanson ont toujours été ses références principales, les influences les plus décisives pour la composition de ses poèmes. D'ailleurs il fait d'une cantatrice de son village sa principale source d'inspiration :

La grande leçon que j'ai reçue de Marône, la poétesse de mon village, est que la poésie est chant sinon musique - et ce n'est pas là un cliché littéraire. Le poème est comme une partition de Jazz, dont l'exécution est aussi importante que le texte. D'un recueil à l'autre cette idée s'est fortifiée en moi ; et lorsqu'en tête d'un poème, je donne une indication instrumentale, ce n'est pas simple formule. Le même poème peut être donc récité - je ne dis pas déclamé - psalmodié ou chanté [...]. Je persiste à penser que le poème n'est accompli que s'il se fait chant, parole et musique en même temps. (Senghor 1990 : 167-168)

À la tête de la plupart de ses poèmes, il donne des indications sur les instruments musicaux qui doivent accompagner leur récitation psalmodique. Certains textes sont désignés par le mot "chant» ou ses équivalences en wolof et en sérère («Woï » et "Guimm », " ndessé »). C'est ce lien assumé avec l'art musical qui explique la place que la couverture accorde à l'image du poète. Sur la pochette du coffret Les rappeurs chantent Senghor (2007), le visage de Senghor, sur un fond noir, couvre toute la surface. $\mathrm{Au}$ bas de sa tête, presque sur le menton, est inscrit le titre du coffret. Senghor est ainsi l'icône (dans tous les sens du mot) centrale de la couverture qui met donc plus l'accent sur lui que sur les rappeurs, suggérant, de ce fait, que sa poésie est l'objet de cet opus. Dans la phrase qui constitue le titre de l'album, son nom joue le rôle grammatical de complément d'objet, signifiant ainsi qu'il est le principal objet de cette création artistique. Toutefois, l'absence d'un nom d'auteur, comme on en trouve sur toutes les couvertures d'album ou de livre, peut signifier que c'est aussi Senghor qui, en quelque sorte, est l'auteur du contenu de cette œuvre (c'est-à-dire ses poèmes). Étant l'auteur de tous les textes qui sont dans le CD à l'exception d'un seul, le chantre de la négritude est à la fois objet et sujet de cette production.

11 Sur un autre plan, l'omniprésence de la couleur noire qui envahit toute la surface de la jaquette, ce visage noir sur un fond noir, s'explique par le besoin de rester fidèle à la manière dont Senghor titrait ses différents recueils qui se réfèrent toujours à cette teinte: Chants d'ombre, Éthiopiques ${ }^{10}$, Hosties noires, Nocturnes. Ce parti pris pour le noir dans la conception de la jaquette est aussi, à notre avis, une référence au mouvement de la négritude. 
12 En outre, si le recto de la pochette, la première page de couverture, renvoie essentiellement à la littérature (avec la figure de Senghor), le verso met en avant la musique. On y trouve plusieurs rubriques: composition musicale, interprète, enregistrement et prise de voix, mixage, mastering, chargé de production, conception jaquette et "bouquin ", etc. On voit là décrite toute la chaîne des participants à la réalisation matérielle de l'œuvre.

\section{Bouquiner avec un CD ou du poème senghorien à l'écriture-image}

Figure 3. Un des textes du livret accompagnant le CD

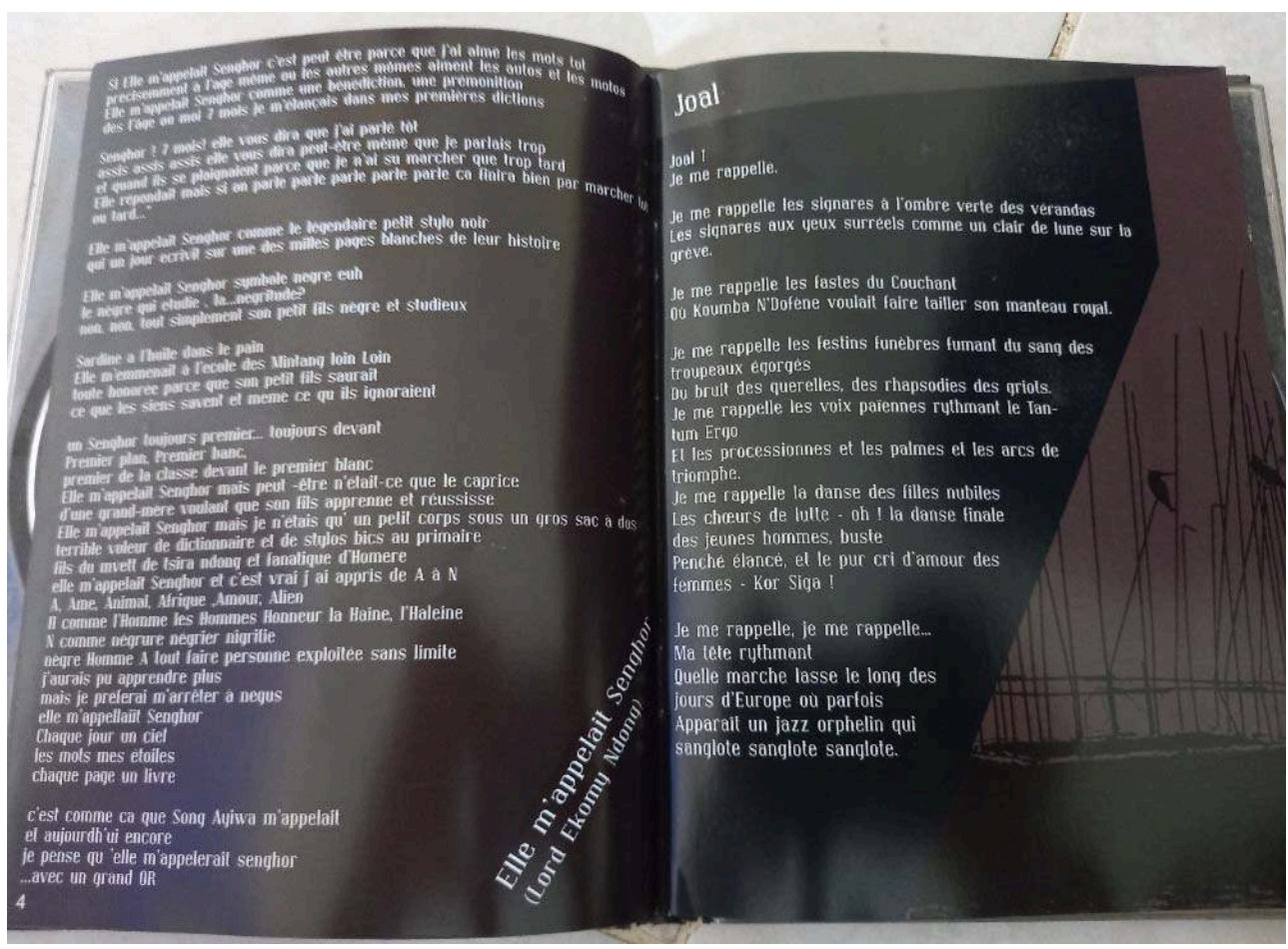

Crédits : Medialab/Waru Studio.

13 L'interaction entre littérature et audiovisuel est matériellement perceptible dès qu'on tient l'opus entre les mains et qu'on commence à le «feuilleter ». Car ici, on a d'abord littéralement un livre de 24 pages, entouré d'un CD et d'un DVD montrant la même image que la pochette. Sur la fiche technique servant de quatrième de couverture, il est mentionné "conception jaquette et bouquin » pour montrer la conscience, chez les producteurs, d'avoir aussi édité un livre. Le livre dont il s'agit ici est numéroté de la page 1 à 24 . Son texte proprement dit commence à la page 2 où se trouve un éditorial (et non une préface). Ce qui nous fait basculer dans un autre média écrit (la presse écrite) ou dans le domaine des supports multimédias où l'on utilise le même terme pour désigner ce texte. Cet éditorial, écrit par le chercheur en littérature orale et musique urbaine Ibrahima Wane, est intitulé "Senghor, musicien de la plume ", titre qui permet de résoudre la contradiction anachronique qui semblait apparaitre dans la nomination de l'album. Par ce titre, l'éditorialiste signale déjà le caractère intermédiatique de l'œuvre senghorienne. 
Cet éditorial, qui prend la place que doit occuper une préface dans un livre, rompt cependant avec la tradition du discours préfaciel tel qu'il apparaît généralement dans les textes littéraires. L'auteur n'y présente pas le contenu de l'œuvre ni les rappeurs participants au projet, ni leurs points de vue ou encore moins le message qu'ils veulent véhiculer. Il se limite à exposer le lien évident entre la poésie de Senghor et la musique. Il ne parle d'ailleurs que de Senghor et de son œuvre, comme pour dire que les rappeurs (dont il devrait normalement parler si nous étions dans une préface classique) ne sont pas, en réalité, les auteurs de l'opus. Ils ne sont pas plus compositeurs de leurs chansons que le poète de la négritude.

Figure 4. Senghor en musique et en image

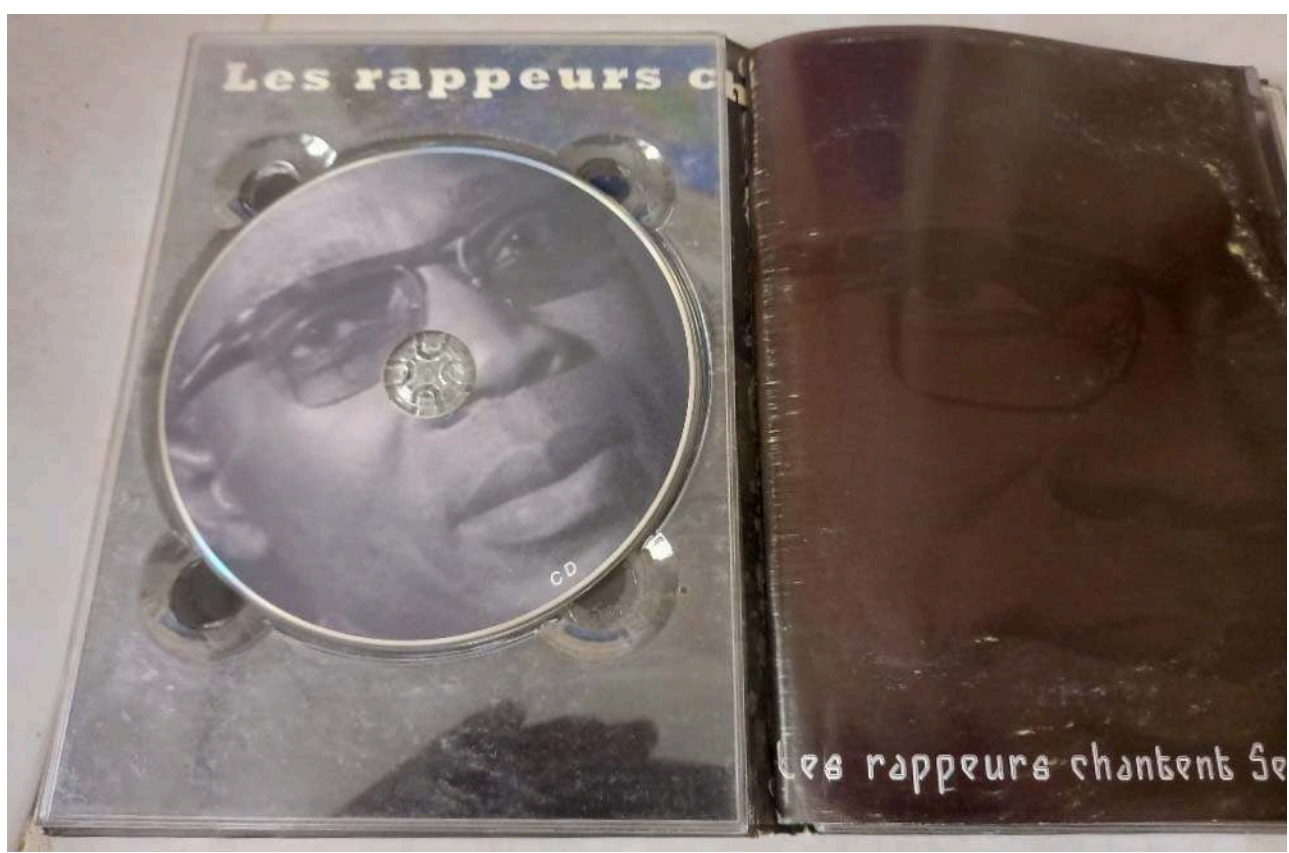

Crédits : Medialab/Waru Studio.

Dans les pages suivantes du livre, sont présentés un à un les rappeurs et les groupes qui ont participé au projet suivant l'ordre qui a été établi par la quatrième de couverture (différente du verso de la pochette). On trouve la photo de chacun des artistes à côté du texte de Senghor qu'ils ont rappé (ou à la page suivante). On verra que ce texte présenté dans le livre (ou «bouquin» comme il est présenté) a parfois de petites différences avec le texte que les rappeurs ont mis en musique. Tous les poèmes sont de Senghor sauf le premier (« elle m'appelait Senghor ») qui est une création originale du rappeur gabonais Lord Ekomy Ndong. Ils sont issus de trois recueils de Senghor : Chants d'ombre (2 textes), Hosties noires ${ }^{11}$ (4) et Éthiopiques (1). La préférence pour les textes de Hosties noires est due, selon notre opinion, au fait que c'est le recueil de Senghor le plus engagé et le plus engageant sur le plan politique, l'auteur y dénonçant les abus et dérives du système colonial que la Seconde Guerre mondiale a exacerbés. Les poèmes choisis ont comme thèmes : la femme africaine, la colonisation, le Royaume d'enfance, la situation des soldats africains pendant la Seconde Guerre mondiale, etc.

Dans ce feuillet, le sujet ou le contenu des textes est illustré par des images photographiques. Ainsi, c'est la photo d'une femme africaine à moitié nue qui se trouve à l'arrière-plan du texte « Femme noire » alors que celle du pont de Joal est convoquée

Itinéraires, 2020-3 | 2021 
pour le poème consacré à cette ville. Le poème "Tirailleur sénégalais » est accompagné de la photo d'un tirailleur noir faisant le salut militaire. Des projectiles d'armes à feu sont utilisés pour "Thiaroye ", dont les vers sont dédiés aux tirailleurs sénégalais massacrés en 1944 par l'armée française dans un camp militaire situé dans la localité du même nom.

Les textes apparaissent, de ce fait, comme des légendes expliquant le contenu des images. Celles-ci sont non seulement des résumés des poèmes mais aussi des éléments visuels s'intégrant dans une œuvre plus globale et sont, de ce fait, érigées en véritable œuvre d'art. On a ainsi une sorte de collage organisé et cohérent différent du collage surréaliste. Parfois le texte est placé sur l'image, comme dans le cas de la page réservée à " Femme noire ", ce qui correspond à un montage sur photo. Cela oblige le récepteur à expérimenter une nouvelle forme de médialité et à faire valoir une compétence polyvalente : interpréter le langage visuel et le langage verbal dans leur simultanéité et leur unité, c'est-à-dire à penser l'image et à voir le texte comme le suggère Bettina Thiers (2012). Ce qui rend possible une transgression et une fusion des arts dans la mesure où il n'y a pas de limite observable entre le texte et l'image qui l'accompagne. Le lecteur peut aussi se limiter à l'image pour avoir une petite idée du contenu du poème, mais sa compréhension ne sera que partielle et fragmentée. Par exemple, celui qui regarde l'icône des projectiles pour le poème "Thiaroye" sait qu'on y évoque quelque chose qui est en relation avec la guerre mais ne saurait exactement de quoi il s'agit réellement que s'il lit le poème. Ce n'est donc pas en réalité l'image qui éclaire la compréhension du texte mais, à l'inverse, c'est le poème de Senghor qui explicite le message visuel.

Dans le livret prennent place également des témoignages d'hommes de culture et de personnalités politiques qui ont eu à cheminer avec Senghor: Djibo Ka (son ancien directeur de cabinet), Amadou Lamine Sall (poète sénégalais vu comme le digne héritier de Senghor dont il fut très proche), Me Boucounta Diallo (avocat de la famille Senghor), Doudou Ndiaye Rose (percussionniste sénégalais de renom), Ibou Diouf (artiste plasticien) et Moustapha Tambédou (conseiller au ministère de la Culture).

Dans le livre, il y a ainsi une cohabitation féconde entre écriture journalistique (éditorial, présentations biographiques d'artistes, témoignages sur une personne disparue) et images photographiques. Nous rappelons que le coffret contient également un DVD, qui n'est pas ici l'objet de notre étude, qui complète l'adaptation visuelle de l'hypotexte senghorien. Ce seul aspect nous fait dire que ce coffret est une œuvre qui illustre véritablement le concept de transmédialité car elle se fonde sur une narration transmédiatique qui est caractérisée par l'usage artistique de plusieurs médias pour développer une expérience unifiée et cohérente. Nous usons ici du terme de narration parce que nous concevons le geste transmédial comme le récit d'une transmission d'un patrimoine littéraire à travers son transfert d'un média à l'autre.

\section{Adaptation musicale, rôle du refrain et réorganisation interne du rythme}

Dans la recherche sur le rap africain, les travaux qui s'intéressent au rythme interne des textes sont presque inexistants. Les études portant sur des aspects intermédiatiques sont rares et semblent souvent ne s'intéresser qu'à la présence de figures de style dans les textes de rap ${ }^{12}$. Or avec ce coffret, il s'agit notamment d'un 
transfert multiple s'opérant par le truchement de la remédiatisation afin de faire migrer le contenu d'une forme médiatique à l'autre. Il s'agit d'abord du passage du poème senghorien du livre au $\mathrm{CD}$ en passant par le livret à travers un savant travail de réaménagement sur la construction rythmique du texte. En mettant en musique les poèmes de Senghor, les rappeurs, tout en restant fidèles à la lettre et à l'esprit des textes, font souvent preuve de créativité dans les rares espaces de liberté qui s'offrent à eux. Ils font preuve d'une audace surtout tournée vers l'organisation rythmique des poèmes et quelquefois vers le contenu du texte lui-même. Conscients que « la scansion d'un texte de rap se caractérise par une dynamique, une impression de mouvement assurée par différents phénomènes rythmiques » (Rubin 2012), Keyti et ses collègues s'adonnent à un travail sur le flow et le rythme afin de réadapter le texte senghorien à la musique rap.

21 Le refrain et le travail sur la musique sont d'autres éléments permettant d'apprécier à sa juste valeur le travail des MCs, qui ne se limitent pas toujours à rendre tel quel le poème senghorien. C'est qu'il y a toujours une différence, même minime, entre le texte écrit et celui qui est effectivement scandé sur la musique par le rappeur. Christian Béthune note, à cet effet, les possibles mutations pouvant s'opérer lors du passage de l'écrit à l'oral :

Il peut en effet y avoir des différences sensibles entre le texte d'un rap imprimé sur le livret d'un album et sa version scandée lors d'un concert, et même entre le texte de la pochette et sa réalisation sonore sur la plage du CD. La prononciation, les accents, les élisions, les apocopes, les syllabes avalées ou au contraire allongées, etc., peuvent différer sensiblement de l'écrit à l'oral, et il ne s'agit pas en l'occurrence de simples modifications de détail puisque c'est toute la perception métrico-rythmique du morceau qui peut se trouver ainsi altérée. (Béthune 2011: 195)

C'est principalement la raison pour laquelle les éléments constitutifs du rythme paraissent avoir la préférence des rappeurs voulant s'adonner à une reprise des textes de Senghor. Pour « une réappropriation orale, rythmique et personnelle d'un matériau poético-musical donné » (Idir 2015), ils focalisent toute leur énergie sur le rythme et l'accentuation qui, constituant les éléments essentiels du style du MC, deviennent ainsi les espaces de renouvellement et de réorganisation du « déjà-là ».

Tout le soin apporté ainsi par un MC comme Keyti à l'organisation rythmique de la chanson, aspect essentiel du rap, prouve les dires de Christian Béthune affirmant que " le rythme occupe une place centrale dans le rap, au point que tous les autres éléments mis en œuvre lui sont en fait subordonnés " (Béthune 2004:64). Le premier de ces éléments demeure le refrain qui est, pour les rappeurs de l'album, le premier espace de liberté où ils peuvent faire preuve de créativité au niveau du rythme. Le seul fait de le mettre dans la chanson est un gage d'originalité car les poèmes de Senghor en sont eux-mêmes rarement pourvus. Ainsi, en reprenant "Lettre à un prisonnier ", Keyti fait du premier vers du poème de Senghor (« Ngom! champion de Thiané ! ») la base du refrain. Ce vers est d'ailleurs traduit en wolof («diambarou Thiané »). Ainsi, cette langue devient, au même titre que le français, une porte d'entrée du texte poétique. Celui-ci devient un espace hétérolingue où l'artiste se fonde sur la traduction afin d'élargir le champ sémantique du mot " champion », le mot wolof utilisé pour le rendre pouvant aussi signifier « héros » ou " personne courageuse ».

Le deuxième vers du poème ( «c'est moi qui te salue, moi ton voisin de village et de cœur») ainsi que le premier de la dernière strophe («Ngom! Réponds-moi par le 
courrier de la lune nouvelle ») complètent le leitmotiv. Le choix de ces deux vers s'explique par leur longueur, qui leur permet de tenir à l'intérieur d'une mesure à quatre temps. Le nom du destinataire, qui est ici interpellé par le procédé de l'apostrophe, comble le vide laissé par la fin du refrain. Il devient une anaphore qui sert de mot d'appui (comme voyelle d'appui) au MC pour relancer le rythme.

Sur le plan sémantique, l'idée de la salutation et celle d'une réponse réclamée par le rappeur procurent à la rengaine la fonction de communication du langage car Keyti tente d'y établir un contact avec le destinataire de son discours. Il fait du refrain un véritable moment de création tout en utilisant uniquement les mots de Senghor. C'est un travail de déconstruction-construction: l'interprète agrège des fragments du texte situés dans des endroits très éloignés pour créer une harmonie sémantique et rythmique à travers le refrain. Le travail de réorganisation rythmique est aussi une œuvre de réinterprétation sémantique qui ajoute au signifié du média d'origine un surplus de sens né de l'imagination du rappeur.

De la même manière, la chanson "Joal» de Xuman s'ouvre sur un refrain. Elle en compte au total trois : au début, au milieu et à la fin. Ce sont les deux premiers vers du poème ( " Joal / Je me rappelle ») qui en sont la base. On a une kora et, en arrière-plan sonore, la voix de la cantatrice sérère Yandé Codou, qui était, au moment où Senghor vivait, une sorte de poétesse officielle du président. Dans la chanson de Yeleen ( Femme noire »), le refrain est chanté en mooré, langue la plus parlée dans le pays des artistes. Pour des besoins d'ordre rythmique, les rappeurs du groupe burkinabé anaphorisent le mot «femme" pour accentuer la fin des différents versets. Quant au rappeur camerounais Boudor, sa première innovation réside dans l'originalité du refrain où il reprend le titre du poème qu'il ressasse en l'accompagnant d'onomatopées à forte consonance africaine afin de mimer des pleurs («Prière de paix / Akibulan na oyé mamé / Prière de paix / Africa mama nia maama makoumbo »).

Les rappeurs s'adonnent à de véritables réaménagements rythmiques en se fondant aussi sur la restructuration du nombre de vers et des couplets. Ainsi, dans le cas de Xuman, le premier couplet du texte qu'il déclame compte six versets ${ }^{13}$ alors que le second en contient neuf. Après chaque verset, il laisse l'instrumental " respirer ${ }^{14}$ » et observe un petit silence avant le vers suivant. Entretemps, c'est souvent la voix lyrique de Yandé Codou qui comble ce vide temporaire. Le nombre de versets du poème oblige le $\mathrm{MC}$ à violer les règles d'organisation du couplet rap qui se construit toujours autour des multiples de quatre. Le premier couplet compte neuf mesures, dix si l'on ajoute celle couverte par le vide verbal laissé après le dernier mot du couplet et avant le refrain. Le deuxième couplet en compte treize ou quatorze.

La déclamation du poème par Xuman adopte ainsi plus le rythme du verset senghorien que celui du morceau de rap classique. Le rappeur montre ainsi une grande fidélité par rapport à la tradition prosodique de la négritude senghorienne tout en opérant de timides changements au niveau du texte.

28 La transition menant du rythme original du poème à celui de la chanson de rap est plutôt réussie par Keyti. Ce dernier ne se sent pas trop gêné par l'organisation rythmique du poème qui s'accorde bien avec son style. Il réussit d'ailleurs, par sa performance, à lui imposer le rythme du break beat en le divisant en différentes mesures hip-hop. Il parvient alors à le condenser, à le rendre plus compact. Sa mesure peut couvrir tout un vers de Senghor et une partie du vers suivant : quand il commence un vers, il ne s'arrête pas à la fin de celui-ci, car il ne se fie pas au rythme recherché par 
le poète, et continue jusqu'au milieu du second vers. Ainsi, rappant sa première mesure, Keyti déclame le deuxième vers mais n'observe pas de pause à sa fin. Il continue jusqu'au mot «blanc» pour observer la pause. Les libertés prises sur la structuration rythmique du poème de Senghor apparaissent si l'on compare les deux œuvres. Si on se fie à la tradition prosodique française et à l'usage de la ponctuation expressive, nous aurons l'organisation rythmique suivante :

C'est moi qui te salue //, moi ton voisin de village et de cœur //.

Je te lance mon salut blanc // comme le cri blanc de l'aurore //, par-dessus les

barbelés //

De la haine et de la sottise //, et je te nomme par ton nom et ton honneur //.

Mon salut au Tamsir Dargui Ndiaye // qui se nourrit de parchemins.

Keyti imprime un tout autre rythme au texte comme le montre l'exemple suivant :

C'est moi qui te salue //, moi ton voisin de village et de cœur. Je te lance mon salut

blanc //

Comme le cri blanc de l'aurore, par-dessus les barbelés de la haine et de la sottise //

Et je te nomme par ton nom et ton honneur $/ /$.

Ngom // Mon salut au Tamsir Daddy Ndiaye. Pluie ${ }^{15}$ de parchemins //.

Par des déplacements de l'accentuation vocalique du vers du poète sérère, Keyti réussit à donner une autre densité accentuelle à la déclamation. De plus, cette reconstruction du rythme du poème senghorien implique la présence de rejets qui n'étaient pas programmés par le poète. Chez Senghor, nous avons, par exemple, «Heureux amis //, qui ignorez les murs de glace et les appartements trop clairs qui stérilisent //Toute graine sur les masques d'ancêtres //et les souvenirs mêmes de l'amour ».

Voici ce que Keyti propose: "Heureux amis, qui ignorez les murs de glace et les appartements trop clairs //Qui stérilisent toute graine sur les masques d'ancêtres et les souvenirs mêmes de l'amour. » Le rejet de la proposition relative au début de la mesure suivante perturbe profondément le rythme du vers senghorien. Le rythme est plus rapide et dense, le rappeur ne laissant pas du tout le beat «respirer ». Il peut aussi décider de ne pas observer une pause qui pourtant s'impose à cause de la ponctuation. Ainsi, avec: «À tous ceux qui, à l'heure où les grands bras sont tristes comme des branches battues de soleil », la pause n'est pas respectée avec la virgule.

Les nombreuses subversions de l'organisation rythmique du texte sont dues au fait que Keyti a voulu resserrer le rythme et accélérer le débit de lecture/déclamation. Il explose la structure métrique d'origine du poème pour le soumettre au rythme à quatre temps du break beat. Ce phénomène est très présent dans les chansons de rap, surtout quand les MCs veulent se départir de la prosodie de la poésie classique. C'est parce que "si la structure métrique du texte explose, la mesure rythmique est toujours là pour structurer la performance [...] dès lors qu'un rythme, c'est-à-dire le retour périodique d'un accent, est assuré par la musique » (Barret 2008 : 124).

Chaque rappeur se donne la liberté de refondre la métrique générale des poèmes et de la réinvestir en usant des possibilités offertes par son propre style qui joue de plusieurs procédés phonétiques allant de l'assonance à l'holorimie. C'est ce qu'ont compris la plupart des interprètes de l'album, comme Boudor, qui structure sa performance rythmique en se focalisant sur une réorganisation de la structure strophique proposée par le poète sénégalais. Sentant qu'il a peu de place pour innover et apporter quelque chose de nouveau au poème senghorien, il concentre toute son énergie sur la répartition des versets dans les différents couplets en divisant le second chant du poème original en deux couplets séparés par le refrain. Il fait subir au poème de 
Senghor une autre organisation séquentielle. Partant d'un poème dont le premier chant comporte neuf vers et le second dix-sept, Boudor aboutit à trois couplets/strophes qui s'organisent ainsi : 9/8/9. Il rend l'organisation du texte plus équilibrée sur le plan de la longueur de ses différentes parties. Là où Senghor part du plus petit (9) au plus grand nombre (17), dessinant ainsi un schéma vertical, le rappeur commence et termine par le plus grand (9) qui encadre le plus petit nombre (8). Ainsi, si nous avons chez Senghor une gradation ascendante ${ }^{16}$ ou crescendo qui augmente la tension rythmique et dramatique, chez le MC camerounais, nous avons une gradation descendante puis ascendante matérialisée par la présence d'une sorte de chute d'abord, puis d'un retour au même niveau et à l'équilibre. Le schéma est ici plus triangulaire que linéaire, plus discontinu que continu. C'est ce qui permet à Boudor, plus fidèle au respect des pauses contenues dans les vers que Keyti, de donner au poème de Senghor un double mouvement contradictoire et une direction multiple.

\section{Le flow, le beat et les autres lieux de la reprise}

Le travail de réaménagement rythmique porte également sur d'autres éléments comme le flow, que Sylvain Berthot définit comme "le phrasé du rappeur, son débit, la maitrise de son souffle et, au-delà, par extension, l'ensemble de son style et de sa technique de rap» (2013: 389). Les flows des rappeurs du projet d'Optimiste Produktions constituent, sans conteste, l'un des instruments de mesure de l'influence du rythme senghorien sur la performance des chanteurs. Ainsi, le flow que Lord Ekomy Ndong débite est plus proche du slam que du rap classique. Cela est dû, peut-être, au fait que le slam est considéré, parmi les arts urbains, comme celui qui est le plus apparenté à la poésie, étant à l'intersection du genre poétique et du rap ${ }^{17}$. En outre, nous notons que la lecture du poème de Senghor modifie sensiblement le flow de Xuman, qui abandonne le rythme à quatre temps du break beat pour se soumettre à celui imposé par l'organisation des vers libres du poète.

De plus, l'accompagnement instrumental des poèmes de Senghor est l'un des constituants de son esthétique. C'est ce qu'ont compris certains beatmakers de l'album. L'instrumental traditionnel utilisé dans la chanson de Xuman plonge l'auditeur mélomane dans un univers typiquement africain que le programmeur et le rappeur réussissent à récréer, conformément au contenu du poème de Senghor. Sont mis en pratique les principes senghoriens de l'enracinement et de l'ouverture qui traversent toute son œuvre. L'affirmation de la culture du continent noir est encore soulignée dans «Elle m'appelait Senghor » de Lord Ekomy Ndong où le break beat est soutenu par le Ngoma, la harpe sacrée du Gabon que joue le rappeur lui-même. Celui-ci, qui a une démarche intermédiale, présente un texte original qu'il a lui-même créé et assume donc le double rôle de lyriciste et de musicien, c'est-à-dire d'auteur, de compositeur et d'interprète. Si cette option est la plus répandue, d'autres chansons font surtout appel, dans leur composition musicale, à un beat très classique. C'est le cas notamment de la chanson de Yeleen.

Concernant les textes déclamés par les rappeurs, l'adaptation des poèmes prend la forme d'une lecture fidèle avec parfois des changements très mineurs. Chez Xuman, deux changements, l'un d'ordre phonologique et l'autre d'aspect lexical, sont notés au niveau du texte : le rappeur prononce [yap.so.diz] au lieu de [yap.so.di] et s'exclame "quelle danse lasse » là où Senghor écrit «quelle marche lasse ». Ce qui lui permet de 
mieux esthétiser le mouvement du corps dont il est question dans ce passage. Ce mouvement n'a plus seulement la fonction utilitaire du déplacement par la marche mais la fonction ludique et toutes les autres que les cultures africaines lui donnent généralement. Par ce petit changement, Xuman transforme un geste banal du quotidien en performance artistique.

Nous notons, avec Keyti, d'autres distorsions du poème de Senghor. En effet, le rappeur ne dit pas «qui se nourrit de parchemin ». Il ne prononce pas «Dargui » mais Daddy. Il prononce le patronyme sénégalais Mbodye (Mbodj) en [mbodzi] à la manière des Français. En outre, le titre du poème n'est pas donné au début comme dans un livre ou dans le «bouquin " du coffret, mais à la fin de la chanson. Il y a donc une différence avec le texte tel qu'il est écrit dans le livret qui accompagne le $C D$, ce qui crée une tension entre l'écrit et l'oral générée par l'opposition du son et de la graphie. La chanson n'est, du reste, pas forcément une lecture fidèle du texte du coffret mais un véritable travail de création rythmique et de réarrangement textuel. Ce qui nous fait dire, contrairement à Christophe Kihm, que la reprise inclut toujours un écart, une transgression pour être un véritable acte de création. Les rappeurs, reprenant les poèmes de Senghor, ont besoin, tout en se soumettant à une fidélité plus ou moins relative, d'observer une certaine distance avec leur modèle. Le rappeur ne donne à la déclamation du texte un cachet original que s'il s'associe au rythme voulu par Senghor en ne se privant pas de le déconstruire de temps en temps. Cyril Vettorato (2013: § 3),qui avance que "le débit des MCs [...] n'existe qu'en s'articulant à d'autres rythmes ", abonde dans le même sens que nous; cela montre l'impératif d'interaction entre le flow $\mathrm{du}$ rappeur et d'autres éléments rythmiques, internes ou externes au texte. C'est là où trouve tout son sens le travail de réaménagement rythmique de Keyti, qui vise à surprendre le lecteur habituel de Senghor en donnant un souffle nouveau au poème de ce dernier. L'actualisation, par le passage d'un média à l'autre, est donc génératrice de nouveauté. Ici, le nouveau peut survenir à travers plusieurs procédés mimant parfois le réel comme avec Boudor. En faisant le choix d'un poème riche en apostrophes et en interpellations, procédés qui abolissent la distance et imposent la proximité, le rappeur camerounais procure à sa chanson le rythme de la conversation.

Quant à Lord Ekomy Ndong, il reprend au début du morceau la première partie d'une célèbre citation de Senghor : «l'émotion est nègre ». Senghor est la source d'inspiration qui lui permet de s'épanouir en réclamant une certaine proximité. Il s'identifie à Senghor et se réclame de la négritude. Le lexique utilisé, les références et les images convoquées dans la chanson la placent dans le sillage des poèmes de la négritude. C'est ce qui apparaît dans le passage suivant :

Elle m'appelait Senghor mais je n'étais qu'un petit corps sous un gros sac à dos / terrible voleur de dictionnaire et de stylos bics au primaire / Fils du mvett de Tsira Ndong et fanatique d'Homère / Elle m'appelait Senghor et c'est vrai j'ai appris de $\mathrm{A}$ à N / A, Âme, Animal, Afrique, Amour, Alien / H comme l'Homme, les Hommes, Honneur, la Haine, l'Haleine / $\mathrm{N}$ comme négrure négrier nigritie / nègre Homme à tout faire personne exploitée sans limite / J'aurais pu apprendre plus / Mais je préférais m'arrêter à Négus / Elle m'appelait Senghor / Chaque jour un ciel / Les mots mes étoiles / Chaque page un livre.

Le rappeur a toujours besoin de retourner à l'objet (a), le poème de Senghor, pour actualiser son œuvre et maintenir le lien avec l'univers de la négritude. Le goût pour les mots, l'attachement à la négritude, à l'Afrique, à sa culture (le mvett), l'ouverture à la culture gréco-romaine avec l'évocation de son admiration pour Homère sont des topoï 
que l'on retrouve également chez Senghor. Leur présence dans la chanson de Ndong participe à la subjectivation du rappeur car, il faut le dire, l'inspiration senghorienne ou la reprise de ses textes, constitue également une opération identitaire ayant un impact aussi bien sur l'œuvre présentée par l'interprète que sur lui-même en tant que sujet. L'acte d'adaptation ne peut donc être sans conséquence sur le rappeur et sur sa musique comme semble le démontrer Christophe Kihm pour qui «les effets de la reprise produisent un mouvement de retour sur l'original, qui le renouvellent, mais aussi des effets de retour sur le sujet, qui le construisent " (Kihm 2010 : 26). L'estime de soi qui, selon Abdoulaye Niang, fait partie des valeurs promues par les rappeurs (Niang 2011), passera donc, au regard du travail de Ndong, forcément par la revalorisation de figures africaines à présenter comme des modèles.

En effet, si les rappeurs réussissent à donner une cure de jouvence aux vers de Senghor et à les rendre accessibles à une jeunesse dont le premier réflexe n'est pas de lire des poèmes de la négritude, ils sont eux-mêmes marqués par ce travail de réadaptation qui leur tient à cœur. L'influence ne se limite donc pas seulement aux mots, au flow et au rythme de leurs raps qui sont soumis à l'organisation interne des poèmes. Kihm semble écrire la même chose quand il affirme que: "Dans cette reconfiguration et cette réorganisation d'une exécution première dans une exécution seconde, se construit le sujet (individu ou groupe)» $(2010: 31)$. C'est moins le sujet que son œuvre qui se construit pour se donner une identité nouvelle.

Ainsi, les chansons qui naissent de ces adaptations de poèmes sont foncièrement différentes de celles qui sont écrites par les rappeurs eux-mêmes, parce que les textes qui constituent la base de ces raps n'ont pas été conçus avec la même intention. Les premiers le sont d'abord pour être déclamés en tant que poèmes, et de ce fait prennent eux-mêmes en charge le volet rythmique et musical, là où les textes écrits par les rappeurs sont conçus pour être accompagnés de ressources externes devant assurer une partie du rythme et de la musicalité.

40 Le volet sonore de ce coffret est donc à la fois répétition, actualisation et renouvellement d'une expérience poétique première, séparée de la seconde par une distance temporelle et une différence de médias, de genres. Par les chansons proposées, les rappeurs réussissent à abolir la distance temporelle et à ressusciter l'œuvre de Senghor. Ainsi, la recréation intermédiatique est une action de renouvellement et de rajeunissement d'une œuvre qui pourrait sembler démodée pour une jeunesse happée par d'autres influences.

41 La belle œuvre artistique Les rappeurs chantent Senghor s'inscrit dans une perspective de variations médiatiques du même qui, passant d'un média à l'autre, d'un support à l'autre, acquiert un supplément de significations que fait percevoir une interprétation polyvalente et intermédiale. On a avec cet opus une narration transmédia dans la mesure où chaque œuvre (le «bouquin », le CD et le DVD) se développe séparément des autres et peut être utilisée et comprise sans se référer au contenu de celles-ci. Cependant, chacune d'elles est produite selon des logiques et des techniques médiatiques différentes. Cela ne les empêche pas pour autant de raconter des histoires qui, tout en prenant la forme du même, s'enrichissent mutuellement dans des sphères matérielles totalement distinctes. Cela signifie que cet opus demande également une réception transmédiale, dans la mesure où l'on est obligé de voir en chacun des médias une partie d'un ensemble indispensable à la compréhension globale de l'œuvre. 
42 Ainsi, ce coffret ne peut être compris dans son ensemble comme œuvre artistique que si l'on fait appel à des outils particuliers favorisant la compréhension du rap en tant que lieu de construction d'un univers narratif, usant des ressources de plusieurs médias afin de raconter l'histoire d'une mise en relation de différents artistes, médias, genres et époques. Cet univers créé par l'interaction entre des médias divers doit faciliter la multiplication des points d'entrée de la chanson rap et de ce coffret en particulier. Ainsi, l'œuvre d'origine, en l'occurrence les poèmes de Senghor, est transférée d'un média à l'autre pour raconter un nouveau récit de la négritude vu de différentes manières.

\section{Conclusion}

L'adaptation musicale des poèmes senghoriens les réactualise et assoit leur contemporanéité. Elle prouve leur caractère protéiforme et leur facilité à s'inscrire sur d'autres supports, posant ainsi la question de la non-irréductibilité de l'œuvre littéraire à sa forme écrite originelle. Appliquant la théorie senghorienne de l'ouverture et de l'enracinement, les différents participants à ce projet témoignent de la porosité de leurs arts respectifs et de leur capacité à s'associer dans une narration transmédiale qui prend toujours appui sur la poésie senghorienne. Cet opus, qui est l'archétype de l'œuvre intermédiatique, est donc un pont entre le mouvement de la négritude et les artistes urbains africains, qui s'inscrivent, de ce fait, dans une tradition littéraire qu'ils essaient d'interroger et d'adapter à leur temps. Le coffret Les rappeurs chantent Senghor peut, in fine être perçu comme un lieu de conservation et de transmission d'une mémoire culturelle panafricaine.

\section{BIBLIOGRAPHIE}

Barret, Julien, 2008, Le rap ou l'artisanat de la rime, Paris, L'Harmattan.

Bertot, Sylvain, 2013, Rap, Hip-Hop : 30 années en 150 albums, Marseille, Le Mot et le reste.

Béthune, Christian, 2004, Pour une esthétique du rap, Paris, Klincksieck.

Béthune, Christian 2011, « Sur les traces du rap », Poétique, n 166, p. 185-201, [En ligne], https:// doi.org/10.3917/poeti.166.0185.

Collectif, 2007, Les rappeurs chantent Senghor, Dakar, Optimiste Produktions (coffret musical).

Idir, 2018, « La théorie rapologique - Flow et versification », Le rap en France, [En ligne, blog], http://johanne.salombo.eu/category/dossiers/lexegese-rapologique/, consulté le 17 février 2021.

Jambrina, Nina, 2015, "Synthèses des travaux de Silvestra Mariniello sur l'intermédialité ", Cinémadoc, [En ligne], http://cinemadoc.hypotheses.org/3097, consulté le 12 novembre 2019. 
Jenkins, Henry, 2006, Convergence Culture. Where old and new media collide, New York, New York University Press.

Kihm, Christophe, 2010, « Typologie de la reprise », Volume, vol. 7, $n^{\circ}$ 1, p. 21-38, [En ligne], https://doi.org/10.4000/volume.887.

Krajewski, Pascal, 2015, « Qu'appelle-t-on un médium ? », Appareil, Articles Varia, [En ligne], https://doi.org/10.4000/appareil.2152.

Mariniello, Silvestra, 2010, «L'intermédialité : un concept polymorphe », dans C. Vieira et I. Rio Novo (dir.), Intermédia. Études en intermédialité, Paris, L'Harmattan, p. 11-29.

Müller, Jürgen, 2016, « Vers l'intermédialité : histoire, positions et options d'un axe de pertinence », Médiamorphoses, $\mathrm{n}^{\circ}$ 16, p. 99-110.

Niang, Abdoulaye, 2011, " "Nous le hip-hop, on le tropicalise" : hip-hop, engagement et renouveau panafricaniste ", CODESRIA, $17^{\mathrm{e}}$ assemblée générale, [En ligne], https//:codesria.org/ general.assembly/IMG/pdf/Abdoulaye_Niang.pdf, consulté le 15 février 2021.

Rubin, Christophe, 2012, « Configurations rythmiques et progression textuelle dans un extrait de rap de Casey ", Revue critique de fixxion française contemporaine, $\mathrm{n}^{\circ}$ 5, [En ligne], http://www.revuecritique-de-fixxion-francaise-contemporaine.org/rcffc/article/view/fx05.11, consulté le 18 février 2021.

Senghor, Léopold Sédar, 1990, Poèmes, Paris, Seuil.

Seye, Serigne, 2020, « Postures hypermédiatiques du rap sénégalais », Intermédialité : dialogue entre les arts et les médias, Revue Sénégalaise de Langues et de Littérature, Nouvelle série, vol. $14, \mathrm{n}^{\circ} 2$, p. 69-85.

Thiers, Bettina, 2012, « Penser l'image, voir le texte. L'intermédialité entre histoire de l'art et littérature », La Vie des idées, 29 juin 2012, [En ligne], http://www.laviedesidees.fr/Penser-1image-voir-le-texte.html, consulté le 12 novembre 2019.

Vettorato, Cyril, 2013, « Le rap ou la démesure de la mesure », Cahiers de littérature orale, $\mathrm{n}^{\circ}$ 73-74, https://doi.org/10.4000/clo.1986.

\section{NOTES}

1. Les hommages aux poètes de la négritude, topos récurrent dans les champs rappologiques français et africain, avec notamment Youssoupha (la chanson « Rendons à Césaire » et l'album Négritude), Abd Al Malik («Césaire » dans l'album Dante), Dinos (le titre «Césaire »), Daara J (citation de Césaire dans « Microphone soldat»), sont surtout tournés vers la figure d'Aimé Césaire. De plus, il n'y a jamais eu de projet consistant à reprendre en rap dans tout un album les textes d'un de ses poètes. Tout cela fait de ce projet une expérience inédite dans le hip-hop. Rappelons aussi qu'en 2001 un groupe de rappeuses avait déjà, lors de la veillée funèbre précédant l'inhumation de Senghor, déclamé en version rap son célèbre poème «Femme noire ». Cependant, le fait de construire tout un album autour des textes de Senghor est un projet original aussi bien dans le rap français que chez les MCs sénégalais.

2. Optimiste Produktions est un label majeur du hip-hop sénégalais et africain dirigé par Safouane Pindra. Ce label, initiateur de la cérémonie des Hip-Hop Awards au Sénégal, avait reçu une importante subvention du gouvernement sénégalais pour ce 
projet, ce qui a rendu possible une distribution gratuite du coffret à la jeunesse africaine via les médiathèques. Cela nous autorise à penser que ce coffret entre dans la droite ligne de la politique culturelle du gouvernement d'Abdoulaye Wade qui revendiquait, malgré son idéologie libérale, l'héritage de Senghor, dont il veut prouver les aspects panafricain et universel.

3. Voir http://leboytown.blogspot.com/2007/10/les-rappeurs-chantent-senghor.html, consulté le 24 janvier 2021.

4. Ce qui est un des objectifs du coffret selon Xuman, l'un des rappeurs du projet, pour qui «c'est un moyen d'inciter la génération Internet qui n'a pas le temps de lire les livres, à revisiter les écrits de Léopold Sédar Senghor inscrits au patrimoine universel » (Ibid.).

5. « Négro-africain » est l'adjectif choisi par les auteurs africains et ceux de la diaspora qui s'étaient réunis au congrès de Paris pour se caractériser. Il sera repris notamment par Lilyan Kesteloot, qui en fait le trait distinctif principal de ces écrivains (Anthologie négro-africaine en 1967 et Histoire de la littérature négro-africaine en 2001).

6. Traduction reprise de Mélanie Bourdaa, «Le transmedia storytelling», Terminal, $\mathrm{n}^{\circ}$ 112, 2013, DOI : https://doi.org/10.4000/terminal.447.

7. Les analyses de cet article doivent aussi beaucoup à notre expérience personnelle de rappeur ayant eu une carrière musicale de quinze ans avant d'embrasser le métier d'enseignant.

8. Genre traditionnel sénégambien assez proche du rap par son caractère scandé. Certains sénégalais pensent d'ailleurs qu'il est le véritable ancêtre du rap.

9. Recueil publié en 1956 et réédité en 1990 par les Éditions du Seuil dans l'ouvrage Poèmes regroupant l'ensemble des recueils poétiques de Senghor. C'est à celui-ci que nous faisons référence dans notre analyse.

10. Les anciens Grecs appelaient "Éthiopiens » les peuples africains de couleur noire vivant au sud de l'Égypte.

11. Recueils publiés respectivement en 1945 et 1948, puis réédités dans le volume de 1990.

12. C'est pour combler ce vide que nous avions publié un article dans une revue sénégalaise pour montrer les différentes possibilités qu'offre une lecture hypermédiatique du rap africain dépassant la seule analyse textuelle: Serigne Seye, «Postures hypermédiatiques du rap sénégalais » (2020).

13. Le mot « verset » est utilisé ici pour faire référence aux vers du poème senghorien qui, très souvent, débordent des limites du vers métrique.

14. Selon l'expression utilisée généralement pas les b.boys sénégalais pour désigner cette partie de l'instrumental où le rappeur ne dit rien, laissant un vide sonore et laissant l'instrumental seul jouer.

15. Le rappeur semble prononcer ici « fruit » au lieu de " pluie ».

16. Gradation qui apparait aussi dans le vers : «car il faut bien que Tu oublies ceux qui ont exporté dix millions de mes fils dans les maladreries de leurs navires / Qui en ont supprimé deux cents millions ».

17. Au Sénégal, le slam est perçu comme une autre branche du mouvement hip-hop parce que les pionniers du slam sont des rappeurs comme Matador de Wa BMG 44, tandis que dans les autres parties du monde, ce sont des poètes qui ont été à l'origine 
de cette tribune d'expression poétique libre. Son inventeur, Marc Kelly Smith, est un poète américain.

\section{RÉSUMÉS}

Cet article rend compte d'une expérience artistique inédite entreprise par des rappeurs africains en 2007. Elle consistait, pour les artistes, à reprendre des poèmes du poète sénégalais Léopold Sédar Senghor afin de les adapter en chanson de rap. Le résultat, un coffret composé d'un livret, d'un $C D$ et d'un DVD, appelle à une réception intermédiale qui prend en compte les multiples rapports implicites et explicites qui lient les médias utilisés (littérature, musique, vidéographie). Dans cet essai, nous allons convoquer les théories de l'intermédialité afin de montrer le travail de recréation multiple que les artistes élaborent sur les poèmes de Senghor.

This paper explores an unprecedented artistic experience initiated by African rappers in 2007. Using the poems written by the Senegalese poet Léopold Sédar Senghor, they adapted them to rap music. This work resulted in the conception of a booklet, a CD and a DVD. It foregrounds an intermedial reception of rap that takes into account the multiple implicit and explicit relations that bind all the media used (literature, music, videography). Building on the theories of intermediality, this essay aims to show multiple processes of re-creation carried out by the artists on Senghor's poems.

\section{INDEX}

Keywords : Senghor (Léopold Sédar), intermediality, African rap, poetry, adaptation, rap artists, transmedia, rap, Africa

Mots-clés : Senghor (Léopold Sédar), intermédialité, rap africain, rap, poésie, adaptation, rappeurs, transmédia, Afrique

\section{AUTEUR}

\section{SERIGNE SEYE}

Université Cheikh Anta Diop de Dakar 